\title{
Counting Magnetic Bipoles on the Sun by Polarity Inversion
}

\author{
Harrison P. Jones ${ }^{1}$ \\ NASA's Goddard Space Flight Center, Laboratory for Astronomy and Solar Physics, \\ Southwest Solar Station, c/o National Solar Observatory ${ }^{\star}$, PO Box 26732, Tucson, \\ AZ 85726, hjones@nso. edu
}

\begin{abstract}
This paper presents a simple and efficient algorithm for deriving images of polarity inversion from NSO/Kitt Peak magnetograms without use of contouring routines and shows by example how these maps depend upon the spatial scale for filtering the raw data. Smaller filtering scales produce many localized closed contours in mixed polarity regions while supergranular and larger filtering scales produce more global patterns. The apparent continuity of an inversion line depends on how the spatial filtering is accomplished, but its shape depends only on scale. The total length of the magnetic polarity inversion contours varies as a power law of the filter scale with fractal dimension of order 1.9. The amplitude but not the exponent of this power-law relation varies with solar activity. The results are compared to similar analyses of areal distributions of bipolar magnetic regions.
\end{abstract}

\section{Introduction}

Loci of polarity inversion for the Sun's line-of-sight magnetic field (commonly but misleadingly termed "neutral lines") have long been a central feature of both observational and theoretical studies of solar activity. Original interest was based on the idea that magnetic reconnection and rapid energy release necessary for impulsive phenomena such as solar flares could only occur in certain polarity configurations near a true null in magnetic field (see, for example, Sturrock, 1968). More recently such regions have been termed separators (Pontin, Priest, and Longcope, 2003). They mark three-dimensional discontinuities in the connectivity of magnetic field lines and only accidentally coincide with polarity inversion. Nonetheless, polarity inversion is still a useful tool for classifying the topography of the Sun's magnetic field and is of considerable interest in, for example, demarcation of coronal structures (McIntosh, 1994), filaments and filament channels (Martin, Bilimoria, and Tracadas, 1993; Chae et al., 2001), and empirical prediction of coronal mass ejections (Falconer, Moore, and Gary, 2002).

Inversion lines are often drawn by hand, left to the eye of the viewer of a designated magnetogram, or are detemined by contouring algorithms with implicitly specified resolution. A more objective, cartographic perspective is taken

- The National Solar Observatory is operated by the Association of Universities for Research in Astronomy under contract with the National Science Foundation. 
for this paper; i.e., focus is on algorithms for determining polarity inversion from magnetograms with pre-specified, explicit resolution scales and on how the character of polarity inversion depends upon this scale.

\section{Method}

The observational data for this paper are magnetograms from the National Solar Observatory/Kitt Peak Vacuum Telescope (NSO/KPVT) (Livingston et al., 1976) which made daily full-disk images of the photospheric line-of-sight field from 1973 to 2003. Incident solar light was passed through a circular polarization modulator and imaged onto a spectrograph entrance slit which subtended a 512 arc-second segment of the solar image. The magnetograph was placed at the exit plane of the astigmatic spectrograph and used the Zeeman wavelength modulation of a spectrum line in circularly polarized light to infer the line-of-sight field at every position along the entrance slit. The solar image was scanned across the entrance slit in a four-swath pattern to form a two-dimensional, full-disk magnetogram.

A recipe for extracting polarity inversion lines from magnetograms is not difficult to devise. The following procedure was especially tuned for application to NSO/KPVT magnetograms.

- Zero-Point Correction: To correct the poorly determined zero point for magnetograms obtained prior to 1992 with the original 512-channel Diode Array Magnetograph, a linear offset was applied for each position of the entrance slit. To compensate for temporal variations of instrument response during the spatial scanning process, the offset amount was computed as the median of all fields at each spatial pixel along the slit within \pm 10 Gauss. (Pixels with these field strengths are representative of the noise distribution of the instrument and should ideally be centered around zero.) Failure to perform this step resulted in polarity inversion contours which close around regions of one polarity.

- Block Averaging: $L \times L$-pixel block averages of the original magnetograms were formed for better continuity of the polarity inversion lines and for enhanced efficiency of spatial filtering. The details of the block averaging enter into the determination of the resolution scale and are discussed in more detail below.

- Smoothing: The block averaged images were further smoothed with a Gaussian filter with standard deviation of $\sigma$ pixels. Again, the details affect the resolution scale. For large images, performing the block average before the Gaussian filter greatly enhances efficiency.

- Algebraic sign: Images were formed of the algebraic sign of the magnetograms after the above two-stage spatial filtering.

- Edge Detection: A change of algebraic sign was detected by comparing the summed absolute values of the four differences between nearest and diagonally nearest pixels to a threshhold. 
Many reasonable variants of this recipe can be constructed which may differ cosmetically, as discussed below, but which will produce the same polarity inversion contours for the same resolution scale. The two-stage smoothing routine used here was developed by trial and error and has two advantages. First, it is efficient since it avoids full Gaussian convolution with large-scale kernels. Second, as discussed below, it enables a simple pixel-counting procedure for measuring the length of the polarity inversion loci without reference to contouring routines.

One can obtain a measure of the scale, $s(L, \sigma)$, by requiring $s$ to be inversely proportional to the the half-width at half-maximum of the Fourier transform of the two-stage block-average/Gaussian smoother (the product of a Gaussian and a sinc function). The constant of proportionality is set by demanding that $s(1,0) \equiv 1$ pixel. Numerical evaluation of this function shows that, within a couple of percent,

$$
s(L, \sigma) \approx L\left(1+10.35 \sigma^{2}\right)^{1 / 2} .
$$

Figure 1 shows inversion line images superposed on original magnetograms for $s=16$ and $s=64$ arc-seconds for two days, one near solar minimum (1984 Sep 15), the other near solar maximum (1992 Dec 15). As the scale decreases, the maps become more complex and outline smaller magnetic features. The maps at a scale of 64 arc-seconds probably best match inversion lines which most researchers would draw.

A measure of this complexity is the total length of the inversion loci. To facilitate measurement of inversion line length, define $n(s, L)$ as the number of inversion-line pixels in an $L \times L$ block-averaged image. If the "width" of the inversion loci were unity, $n L$ would measure the length in pixels of the original image. Emperically,

$$
\operatorname{Ln}(s, L) \approx \text { constant } \equiv n(s, 1)
$$

for a subrange of $L$ at a given $s$ but falls below this relation as $L$ approaches unity where the inversion lines lose continuity. Thus one may determine the "length" of the inversion loci in pixel units of the original image by extrapolation to unit pixel scale and averaging over the subrange:

$$
\ell(s) \equiv n(s, 1) \approx\langle\operatorname{Ln}(s, L)\rangle .
$$

\section{Analysis}

Inspection of logarithmic plots of $\ell(s, L)$ shows a power-law (fractal) dependence with respect to scale over nearly two orders of magnitude of scale. Since this behavior is reminiscent of the areal distributions reported for bipolar magnetic regions by Harvey and Zwaan (1993), $\ell(s, L)$ has been computed for their sample of of 233 active-Sun and 259 quiet-Sun magnetograms. The results are shown in Figure 2. Solid lines connect the average data, dashed lines are the linear least-squares fits to the $\log$-log plots, and "error" bars show \pm one standard 

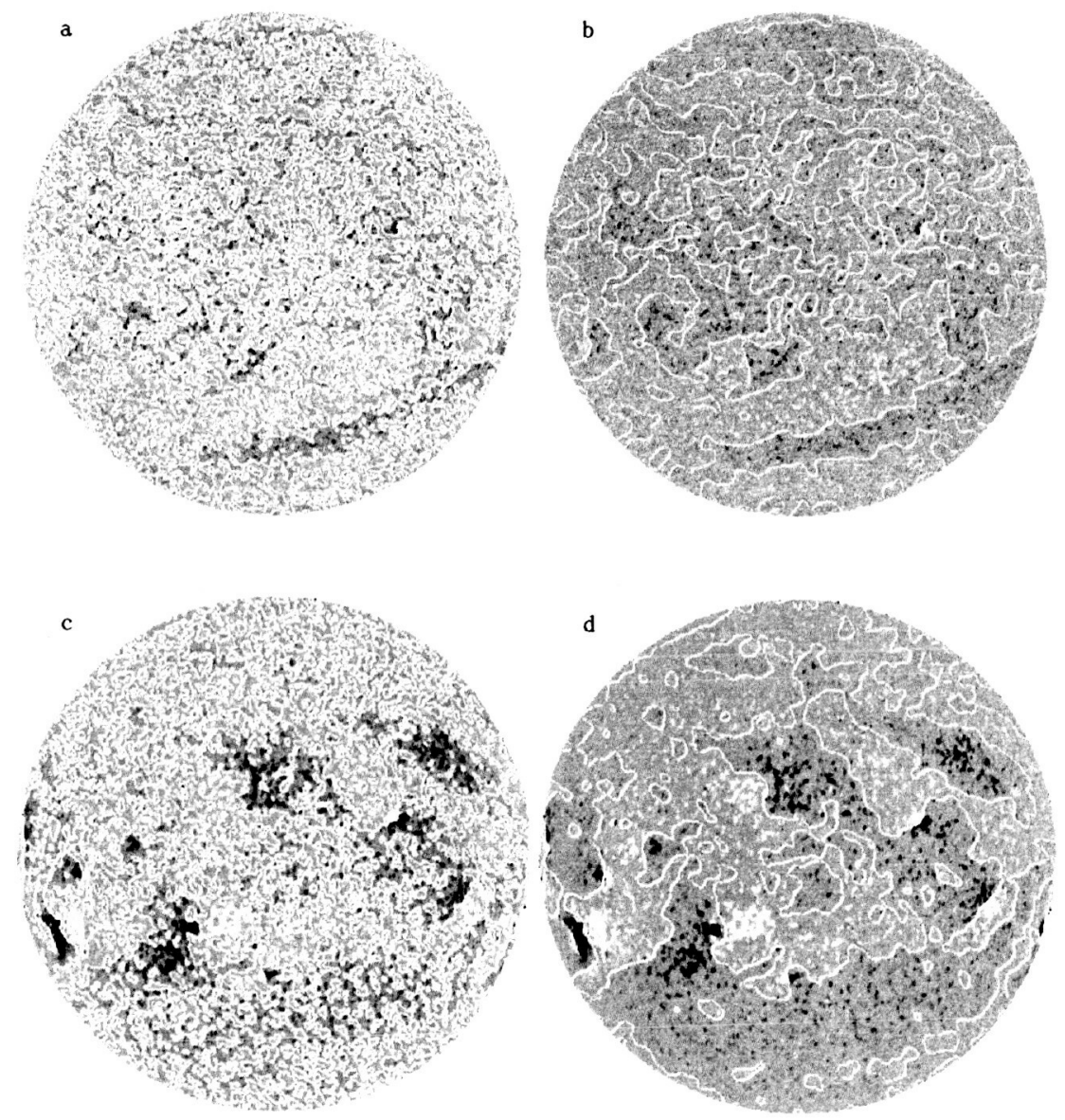

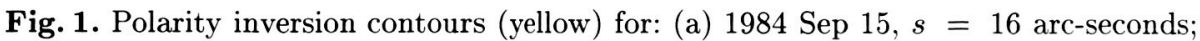
(b) 1984 Sep 15, $s=64$ arcseconds; (c) 1992 Dec 12, $s=16$ arc-seconds; (d) 1992 Dec $12, s=64$ arcseconds. $L=4$ pixels for all panels. 
deviation (not standard error of the mean) over the respective samples. The fractal dimensions of the linear fits are $-1.873 \pm 0.005$ and $-1.891 \pm 0.008$ for quiet- and active-sun magnetograms, respectively. Uncertainties here are \pm 3 standard errors of the mean of individual fits over the entire sample. The log amplitudes at 0.033 solar radii are $2.220 \pm 0.003$ and $2.129 \pm 0.005$ respectively.

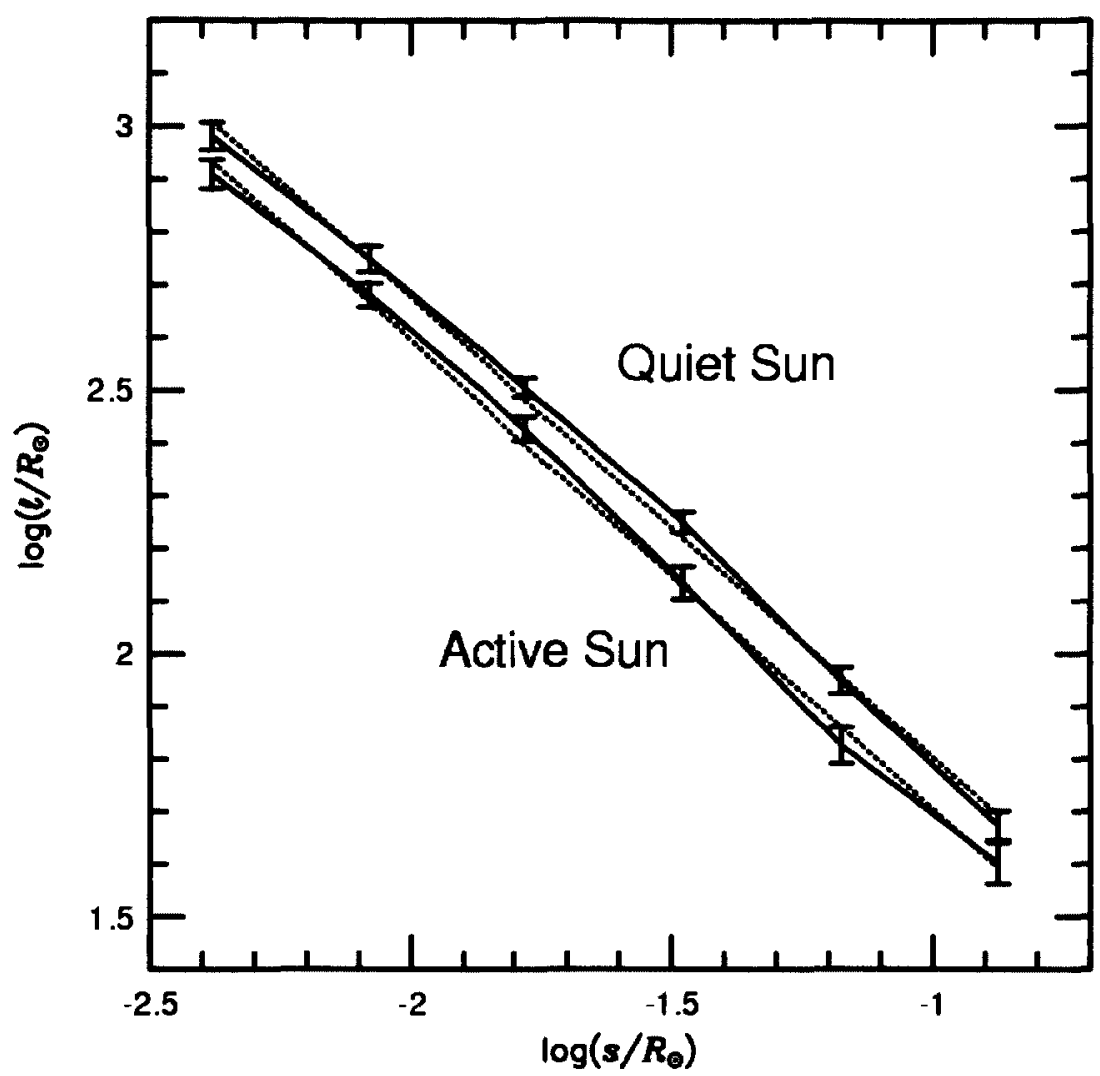

Fig. 2. Inversion line length vs resolution scale for the active- and quiet- magnetogram samples of Harvey and Zwann (1993). Solid lines connect average data points with sample standard deviations indicated by error bars. Dashed lines show linear fits to the averages.

Note from Fig. 2 that the amplitude of the variation varies with solar cycle, but the exponent of the power law does not, a behavior similar to that found by Harvey and Zwaan (1993). To compare with their results in more detail, one may characterize their results as

$$
n(t, A) \sim f(t) g(A) .
$$


One finds from their Figure 4 that $f($ active $) / f($ quiet $) \approx 5$ and $g(A) \propto A^{-1.8}$. From the inversion line results, $\ell_{\text {quiet }}(s) / \ell_{\text {active }}(s) \simeq 1.23$. By expressing inversion line length as a function of resolution area rather than scale, one can combine these results to infer an emperical relation:

$$
\ell(s) \sim \text { const } *\left[g\left(s^{2}\right) / \sqrt{f(t)}\right]^{0.25} .
$$

Thus, at least empirically, length of the polarity inversion loci may be used to "count" bipolar magnetic regions.

\section{Discussion}

This paper outlines simple procedures for efficiently smoothing magnetograms with a well-defined and pre-specified resolution scale and deriving loci of polarity inversion from the smoothed magnetograms. Unlike subjective methods or packaged contouring routines, the resolution scale is both objective and fixed. A reasonable measure of inversion-line length varies as a power-law of resolution scale over about two orders of magnitude with a fractal dimension of about 1.9, irrespective of solar activity; that is, inversion lines are nearly space-filling. Finally, by comparing the results to the statistics for bipolar regions derived by Harvey and Zwaan (1993) an approximate relation is inferred which suggests that polarity inversion is an economical way to measure the areal distribution of bipolar magnetic regions.

Future work, includes extending the analysis to all available magnetograms from the NSO/KPVT and comparing the results with simulations and physical models of solar magnetic fields. Is this result a constraining feature of such models or does it inevitably apply to a wide class of models?

\section{Acknowledgements}

NSO/KPVT data used in this paper were produced cooperatively by NSF/AURA, NASA's GSFC, and NOAA/SEC. This work was supported by NASA Office of Space Sciences grants 344-12-51-14 and 344-12-51-19.

\section{References}

Chae, J., Martin, S. F., Yun, H. S., Kim, J., Lee, S., Goode, P. R., Spirock, T., and Wang, H.: Small Magnetic Bipoles Emerging in a Filament Channel. Astrophys. J. 548 (2001) 497-507.

Falconer, D. A., Moore, R. L., Gary, G. A.: Correlation of the Coronal Mass Ejection Productivity of Solar Active Regions with Measures of Their Global Nonpotentiality from Vector Magnetograms: Baseline Results. Astrophys. J. 569 (2002) 1016-1025.

Harvey, K. L. and Zwaan, C.: Properties and Emergence of Bipolar Active Rregions. Solar Phys. 148 (1993) 85-118. 
Livingston, W. C., Harvey, J., Pierce, A. K., Schrage, D., Gillespie, B., Simmons, J., Slaughter, C.: The Kitt Peak 60-cm Vacuum Telescope. Appl. Opt. 15 (1976) 33-39.

Martin, S. F., Bilimoria, R., and Tracadas, P. W.: Magnetic Field Configurations Basic to Filament Channels and Filaments. Bull. Amer. Astron. Soc. 25 (1993) 1217.

McIntosh, P. S.: YOHKOH X-Ray Image Interpretation with Overlays of $\mathrm{H} \alpha$ Neutral Lines. in X-ray Solar Physics from Yohkoh, Y. Uchida, T. Watanabe, K. Shibata, and H. S. Hudson (eds.) (1994) 271.

Pontin, D. I., Priest, E. R., and Longcope, D. W.: A Framework for Understanding the Topology of Complex Coronal Structures. Solar Phys. 212 (2003) 319-342.

Sturrock, P. A.: A Model of Solar Flares. in Structure and Development of Solar Active Regions, K. O. Kiepenhuer (ed.), IAU Symposium 35 (1968) 471-479. 